\title{
Dehalococcoides as a Potential Biomarker Evidence for Uncharacterized Organohalides in Environmental Samples
}

\author{
Qihong Lu't, Ling Yu't, Zhiwei Liang', Qingyun Yan', Zhili He', Tiangang Luan², \\ Dawei Liang ${ }^{3 *}$ and Shanquan Wang ${ }^{1,4 *}$
}

${ }^{1}$ Environmental Microbiome Research Center and the School of Environmental Science and Engineering, Sun Yat-sen University, Guangzhou, China, ${ }^{2}$ State Key Laboratory of Pest Control and Resource Utilization, School of Life Sciences, Sun Yat-sen University, Guangzhou, China, ${ }^{3}$ Beijing Key Laboratory of Bio-inspired Energy Materials and Devices, School of Chemistry and Environment, Beihang University, Beijing, China, ${ }^{4}$ Guangdong Provincial Key Laboratory of Environmental Pollution Control and Remediation Technology, Guangzhou, China

The massive production and improper disposal of organohalides resulted in worldwide contamination in soil and water. However, their environmental survey based on chromatographic methods was hindered by challenges in testing the extremely wide variety of organohalides. Dehalococcoides as obligate organohalide-respiring bacteria exclusively use organohalides as electron acceptors to support their growth, of which the presence could be coupled with organohalides and, therefore, could be employed as a biomarker of the organohalide pollution. In this study, Dehalococcoides was screened in various samples of bioreactors and subsurface environments, showing the wide distribution of Dehalococcoides in sludge and sediment. Further laboratory cultivation confirmed the dechlorination activities of those Dehalococcoides. Among those samples, Dehalococcoides accounting for $1.8 \%$ of the total microbial community was found in an anaerobic granular sludge sample collected from a full-scale bioreactor treating petroleum wastewater. Experimental evidence suggested that the influent wastewater in the bioreactor contained bromomethane which support the growth of Dehalococcoides. This study demonstrated that Dehalococcoides could be employed as a promising biomarker to test the present of organohalides in wastestreams or other environmental samples.

Keywords: Dehalococcoides, biomarker, environmental samples, organohalide compounds, reductive dehalogenation

\section{INTRODUCTION}

Organohalide compounds are a giant group of halogen-substituted hydrocarbons produced in large quantities as solvents, plastics, pesticides, and chemical intermediates for industrial and agricultural uses (Stringer and Johnston, 2001; Jugder et al., 2016). The improper handling and disposal of harmful halogenated compounds resulted in their worldwide contamination in soil and water as well as bioaccumulation through food webs, posing threat to both human health and the environment (Stringer and Johnston, 2001; Zhou et al., 2004; Lu et al., 2017). Due to 
the side effects on biota, 69 out of the 126 EPA Priority Pollutants are organohalide compounds (United States Environmental Protection Agency, 2013). However, detection and monitoring of their environmental transport and fate using chromatographybased methods were limited due to the extremely wide variety of organohalide compounds (Stringer and Johnston, 2001).

Anoxic aquatic sediments became the major environmental sink for hydrophobic organohalide compounds, facilitating the growth of dehalogenating bacteria through organohaliderespiration (Smidt and de Vos, 2004; Zhou and Song, 2004; Rossi et al., 2012). In the organohalide-respiration process, anaerobic bacteria couple their growth with halogen-removal using acetate as a carbon source, $\mathrm{H}_{2}$ as an electron donor, and various organohalides as electron acceptors (Mohn and Tiedje, 1992; Holliger and Schumacher, 1994). Thus far, phylogenetically diverse bacterial groups have been identified to be able to remove halogens from organohalide compounds, including Dehalococcoides, Dehalogenimonas, Dehalobium, Dehalobacter and Desulfitobacterium (Smidt and de Vos, 2004; Zanaroli et al., 2015; Wang et al., 2016), which were normally originated from contaminated sites (Hendrickson et al., 2002; Taş et al., 2009; van der Zaan et al., 2010). Among them, Dehalococcoides are obligate organohalide-respiring bacteria that exclusively employ acetate as a carbon source, $\mathrm{H}_{2}$ as an electron donor and organohalides as electron acceptors to conserve energy for growth (Löffler et al., 2013). Dehalococcoides were identified to have the most diverse and extensive dehalogenation activities on organohalide compounds, including chloroethenes (MaymóGatell et al., 1997; He et al., 2003; Müller et al., 2004), chlorobenzenes (Adrian et al., 2000), polychlorinated biphenyls (PCBs) (Bedard et al., 2007; Wang et al., 2014), polybrominated diphenyl ethers (PBDEs) (He et al., 2006), chloroethanes and chlorophenols (Fennell et al., 2004; Lookman et al., 2004; Adrian et al., 2007; Wang and He, 2013a,b). Therefore, Dehalococcoides might be employed as a potential biomarker, complementing current chromatography-based methods, to test the presence of organohalide compounds.

In this study, we first screened Dehalococcoides in sludge and sediment samples collected from various anaerobic bioreactors for industrial wastewater treatment and contaminated blackodorous urban rivers. Further source-tracking together with laboratory cultivation confirmed which organohalide compounds supported the growth of Dehalococcoides. These results opened up opportunities employing Dehalococcoides as a biomarker to track unknown sources of organohalide compounds in wastewater and environmental samples.

\section{MATERIALS AND METHODS}

\section{Microbial Cultures Setup and Transfer}

Sludge and sediment samples collected from bioreactors and black-odorous urban rivers were employed as inoculum for culture setup (Table 1). These samples were acquired directly by filling sterile $50 \mathrm{ml}$ plastic Falcon tubes that were capped and transported to the laboratory at an ambient temperature. To control exposure of the samples to oxygen, Falcon tubes were sealed with Parafilm, and microcosm setup was performed in anaerobic chamber soon after their arrivals. For granular sludge, it was smashed into floc-form sludge before inoculation. Defined anaerobic mineral medium in $160 \mathrm{ml}$ serum bottles for microbial cultivation was prepared as described (He et al., 2003; Wang and He, 2013a), which contains salts, trace elements and vitamins. L-cysteine and $\mathrm{Na}_{2} \mathrm{~S} \cdot 9 \mathrm{H}_{2} \mathrm{O}(0.2 \mathrm{mM}$ each $)$ were added to the medium to achieved reduced conditions. The bottles were sealed with black butyl rubber septa and secured with aluminum crimp caps. The organohalide-fed cultures were transferred in $100 \mathrm{ml}$ medium supplemented with $10 \mathrm{mM}$ lactate, $10 \mathrm{mM}$ 2-bromoethanesulphonate (BES, to inhibit methanogen growth), and $1 \mathrm{mM}$ PCE or $10 \mathrm{ppm}$ chloromethane. The control cultures without organohalide-amendment were transferred in the same mineral medium. Unless stated otherwise, cultures were incubated at $30^{\circ} \mathrm{C}$ in the dark without shaking. All the experiments were set up in duplicates.

\section{Analytical Techniques}

Headspace samples of chloroethenes (i.e., PCE, TCE, cis-DCE, trans-DCE, VC and ethane)and chloromethane were injected manually with a glass, gastight, luer lock syringe (Hamilton, Reno, NV, United States) into a gas chromatography (GC) 7890N equipped with a flame ionization detector (Agilent, Wilmington, DE, United States) and a GS-GasPro column $(30 \mathrm{~m} \times 0.32 \mathrm{~mm}$; Agilent, Wilmington, DE, United States) as described (Wang and $\mathrm{He}, 2013 \mathrm{~b}$ ). The standards compounds (with analytical pure or above) were purchased from Sigma-Aldrich.

\section{Fluorescence In Situ Hybridization (FISH)}

The FISH experiment was performed according to protocols described previously (Amann et al., 1995). Granular sludge samples were fixed in a $4 \%$ paraformaldehyde solution for $8 \mathrm{~h}$ at $4^{\circ} \mathrm{C}$, and embedded in Optimal Cutting Temperature (O.C.T.) compound (Fisher Healthcare, Houston, TX, United States). Then the freezing granules were cut into $15 \mu \mathrm{m}$-thick sections with CM3050S cryostat (Leica, Germany). Hybridization was performed at $46^{\circ} \mathrm{C}$ for $4 \mathrm{~h}$ with oligonucleotide probes Dhe1259 (Yang and Zeyer, 2003), EUBmix and ARCH915 (Amann et al., 1995) targeting Dehalococcoides, bacteria and archaea, respectively. Dhe1259 and EUBmix/ARCH915 for dual-staining FISH were labeled with Cyanine 3 (Cy3) and Cy5, respectively. FISH-stained images were captured CLSM (Leica TCS-SP2, Germany).

\section{DNA Extraction, PCR, and Illumina Miseq Sequencing}

Community gDNA was extracted using the FastDNA Spin Kit for Soil (MP Biomedicals, Carlsbad, CA, United States) according to the manufacturer's instructions. The $16 \mathrm{~S}$ rRNA gene was amplified with the U515F forward primer and U909R reverse primer as described (Narihiro et al., 2015). Illumina Miseq sequencing (Illumina, San Diego, CA, United States) service was provided by BGI (Shenzhen, China). The provided pairend $(2 \times 300$ nd) demultiplexed sequences were assembled and filtered using Mothur v.1.33 (Schloss et al., 2009). 
TABLE 1 | Sludge samples information which collected from anaerobic industrial wastewater treating bioreactors and environmental samples.

\begin{tabular}{|c|c|c|c|c|c|}
\hline Sample No. & Sludge/sediments source & Sludge/sediments Form & Bioreactor type & Dehalococcoides occurrence & Dechlorination activity \\
\hline 1 & Vitamin-C Industry & Granules & UASB & - & - \\
\hline 2 & Petrochemical Industry & Granules & UASB & + & + \\
\hline 3 & Brewery Industry & Granules & UASB & - & - \\
\hline 4 & Paper mill Industry & Granules & UASB & - & - \\
\hline 5 & Coke Industry & Flocs & Anaerobic digester & - & - \\
\hline 6 & Acrylic textile Industry & Flocs & Anaerobic digester & - & - \\
\hline 7 & Textile-dyeing Industry & Flocs & Anaerobic digester & - & - \\
\hline 8 & WAS Anaerobic digestion Industry & Flocs & Anaerobic digester & - & - \\
\hline 9 & Black-odorous River A & Flocs & N.A. & + & + \\
\hline 10 & Black-odorous River B & Flocs & N.A. & + & + \\
\hline 11 & Black-odorous River C & Flocs & N.A. & + & + \\
\hline
\end{tabular}

Quantitative Insights Into Microbial Ecology (QIIME, v1.8.0) was employed for the subsequent processing and downstream analysis (Caporaso et al., 2010).

\section{Data Deposition}

Raw Illumina Miseq sequencing reads were deposited into NCBI Sequence Read Archive (SRA) with accession no. SRP112682.

\section{RESULTS}

\section{Screening of Obligate Organohalide-Respiring Dehalococcoides in Anaerobic Sludge and Sediment Samples}

Dehalococcoides as an obligate dehalogenating bacterial group can only utilize organohalides as electron acceptors to support their growth (Löffler et al., 2013). In this study, sediment and sludge samples from black-odorous urban rivers and anaerobic bioreactors, respectively, were selected to screen the presence of Dehalococcoides (Table 1). PCR amplification with Dehalococcoides genus-specific primers, FpDHC1/RpDHC1377 (Hendrickson et al., 2002), showed the positive detection of Dehalococcoides in all urban river sediment samples, as well as in a granular sludge sample collected from a full-scale mesophilic UASB reactor treating petrochemical wastewater (Table 1). And the petrochemical wastewater contains organic compounds generated from terephthalic-acid industry, e.g., terephthalic-acid, benzoic acid, toluic acid, acetic acid and other intermediate compounds and byproducts (Lykidis et al., 2011).

To profile microbial communities of those Dehalococcoidescontaining environmental samples, Miseq 16S rRNA gene sequencing was performed, showed the very different microbial community structure in samples between Dehalococcoidescontaining granular sludge and urban river sediments (Figure 1). In granular sludge collected from the UASB reactor, acidogenic populations, Syntrophorhabdus (of Syntrophorhabdaceae) and Syntrophus, formed syntrophic interactions with methanogenic Methanosaeta and Methanosarcinaceae (Figure 1). Surprisingly, the obligate organohalide-respiring Dehalococcoides presented abundant in the full-scale UASB reactor, accounting for $1.83 \%$ of the total microbial community, comparable with the relative abundance of Dehalococcoides in enrichment cultures dechlorinating PCBs (Wang and He, 2013a) and PCE (Lee et al., 2015). The presence of abundant obligate organohaliderespiring Dehalococcoides implied that the TA-wastewater contained uncharacterized organohalide compound(s). In the UASB reactor, acetate and $\mathrm{H}_{2}$ generated from degradation of aromatic compounds in petrochemical wastewater by Syntrophorhabdus, Syntrophus and other syntrophs, together with low redox potential and the uncharacterized organohalide compounds, provide ideal growth niches for the fastidious Dehalococcoides. No other obligate dechlorinating bacteria, e.g., Dehalogenimonas and Dehalobacter, were found in the granular sludge sample. In a control sample collected from a lab-scale anaerobic sludge digester without organohalide amendment, no known dechlorinating bacteria can be detected (Figure 1). The highly similar microbial community structures of the three black-odorous river sediments, distinguish themselves from the community compositions of the granular sludge, especially the predominant lineages of Chloroflexi (i.e., Longilinea, GCA004, WCHB1-05 and Anaerolinaceae) and Proteobacteria (i.e., Syntrophobacter and Dechloromonas) (Şimsir et al., 2017) (Figure 1). Dehalococcoides were shown the appearance in the microbial community, on which indicate the potential of organohalides' contamination.

\section{Dechlorination Activities in Dehalococcoides-Containing Cultures}

To further evaluate the dechlorination activities, perchloroethene (PCE) was spiked into microcosms established with those Dehalococcoides-containing sediment and sludge samples. After around 2 months' incubation, PCE dechlorination activities were observed in all three microcosms with the river sediment inocula (data not shown). Subsequent consecutive culture transfers of the three microcosms generated three active cultures which reductively dechlorinate PCE into vinyl chloride (VC) or ethene (Figure 2). No dechlorination activity was observed in the control microcosm established with digester sludge (Figure 2D).

In contrast to PCE dechlorination in sediments of the three black-odorous urban rivers, microcosms inoculated with the 


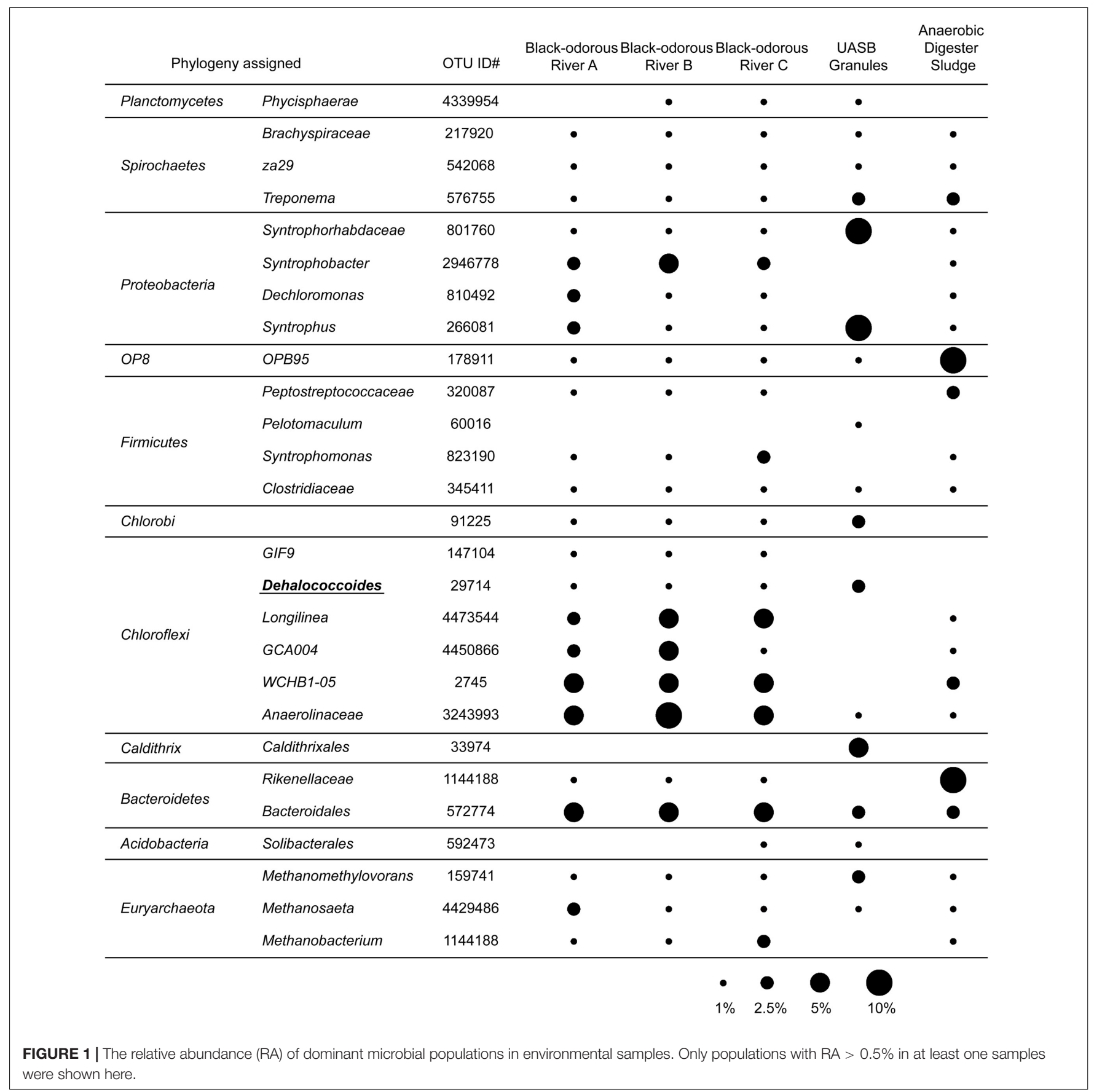

Dehalococcoides-containing granular sludge showed negative PCE-dechlorination activity. To identify potential organohalides to support the growth of Dehalococcoides in the granular sludge, organohalide pollution in the petrochemical wastewater as influent of the UASB reactor was evaluated. The petrochemical wastewater was generated from a AMOCO process that oxidize para-xylene to terephthalic-acid, using a homogeneous catalyst of cobalt and manganese together with bromide as a promoter, in which bromomethane was generated as a byproduct (Tomás et al., 2013). Due to difficulties in obtaining bromomethane, dehalogenation activity test was performed with chloromethane as a homolog alternative to bromomethane. In chloromethanefed culture, over $70 \%$ chloromethane was dechlorinated within 8 days (Figure 3). No obvious dechlorination activity was observed in abiotic control.

\section{Dehalococcoides in the Granular Sludge}

The partial 16S rRNA gene sequences ( $\sim 400 \mathrm{bp}$ ) generated from Miseq sequencing of V4-V5 hypervariable regions were unable to differentiate Dehalococcoides between Cornell and Victoria subgroups. Therefore, Dehalococcoides genus-specific primers (i.e., FpDHC1/RpDHC1377) were utilized to generate longer $16 \mathrm{~S}$ 


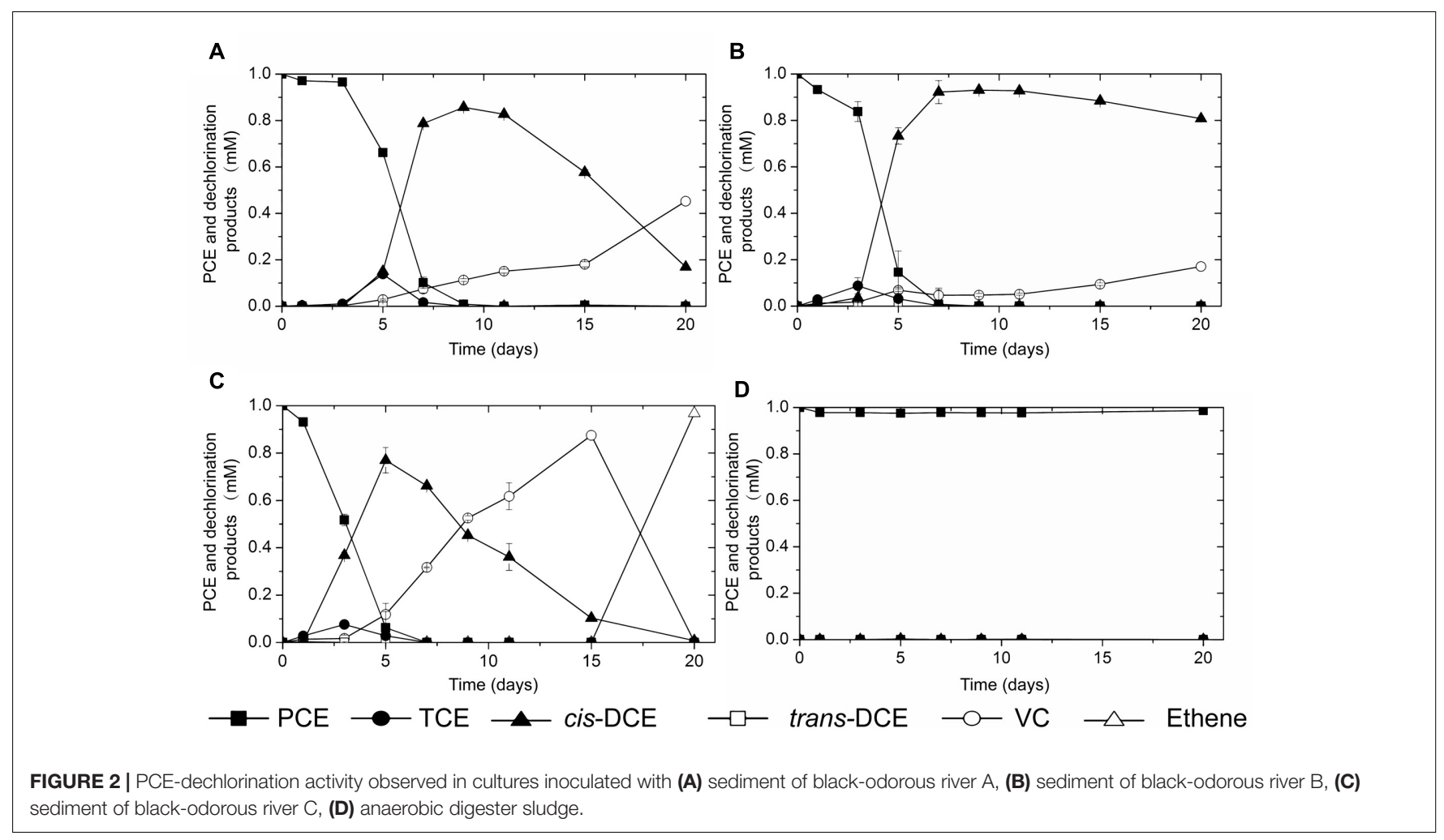

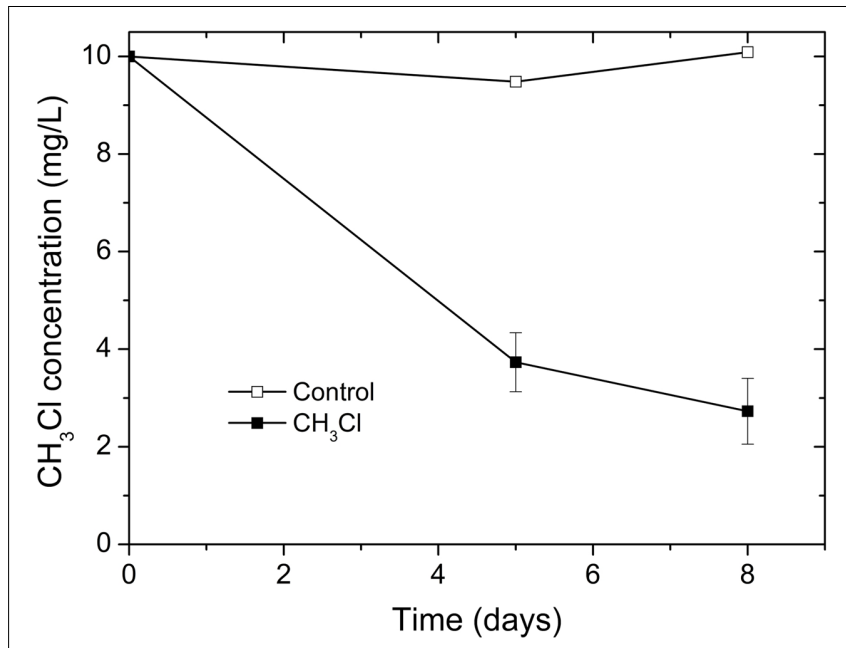

FIGURE 3 | Dechlorination of chloromethane by UASB granules.

rRNA gene sequences $(\sim 1300 \mathrm{bp})$ to identify the Dehalococcoides in the anaerobic granular sludge. Phylogenetic analysis showed the close clustering of Dehalococcoides in TA-degrading granules with $D$. mccartyi 195 in Cornell subgroup (Figure 4A), sharing 99\% 16S rRNA gene sequence similarity (2 bp difference over $1311 \mathrm{bp}$ ) with that of strain 195.

To provide insight into the spatial distribution of Dehalococcoides in the granular sludge, FISH was conducted with Dehalococcoides-specific, bacterial and archaeal oligonucleotide probes (Amann et al., 1995; Yang and Zeyer, 2003). FISH analysis showed the scattered distribution of Dehalococcoides inside granules, closely colonized with other bacteria (Figure 4B) but separated from archaea (Figure 4C). Degradation of aromatic compounds by fermentative bacteria is thermodynamically restricted and will become endergonic $(\Delta G>0)$ as metabolic byproducts (e.g., acetate and $\mathrm{H}_{2}$ ) accumulate in the biosystem. Similar with methanogenic archaea, Dehalococcoides might form syntrophic interactions with aromatic compound degrading acidogens in the granular sludge: the degradation of aromatic compounds by Syntrophorhabdus and other syntrophs provide acetate as carbon source and $\mathrm{H}_{2}$ as electron donor for the halorespiration of Dehalococcoides; correspondingly, Dehalococcoides help maintain acetate and $\mathrm{H}_{2}$ at low concentration in the biosystem and 'pull' degradation of aromatic compounds toward completion through consuming metabolic byproducts generated by acidogenic bacteria. The close colonization of Dehalococcoides with syntrophic bacteria could facilitate the interspecies transfer of $\mathrm{H}_{2}$ (Mao et al., 2015).

\section{DISCUSSION}

Thus far, it remains challenging to detect organohalide compounds in wastewater and environmental samples based on chromatography methods due to their extremely wide variety, e.g., PCBs are a family of 209 structurally similar congeners (Chu and Hong, 2004; Elder et al., 2008). Bromomethane, similar with many other organohalide compounds produced as intermediate or byproducts in chemical synthesis processes, was 

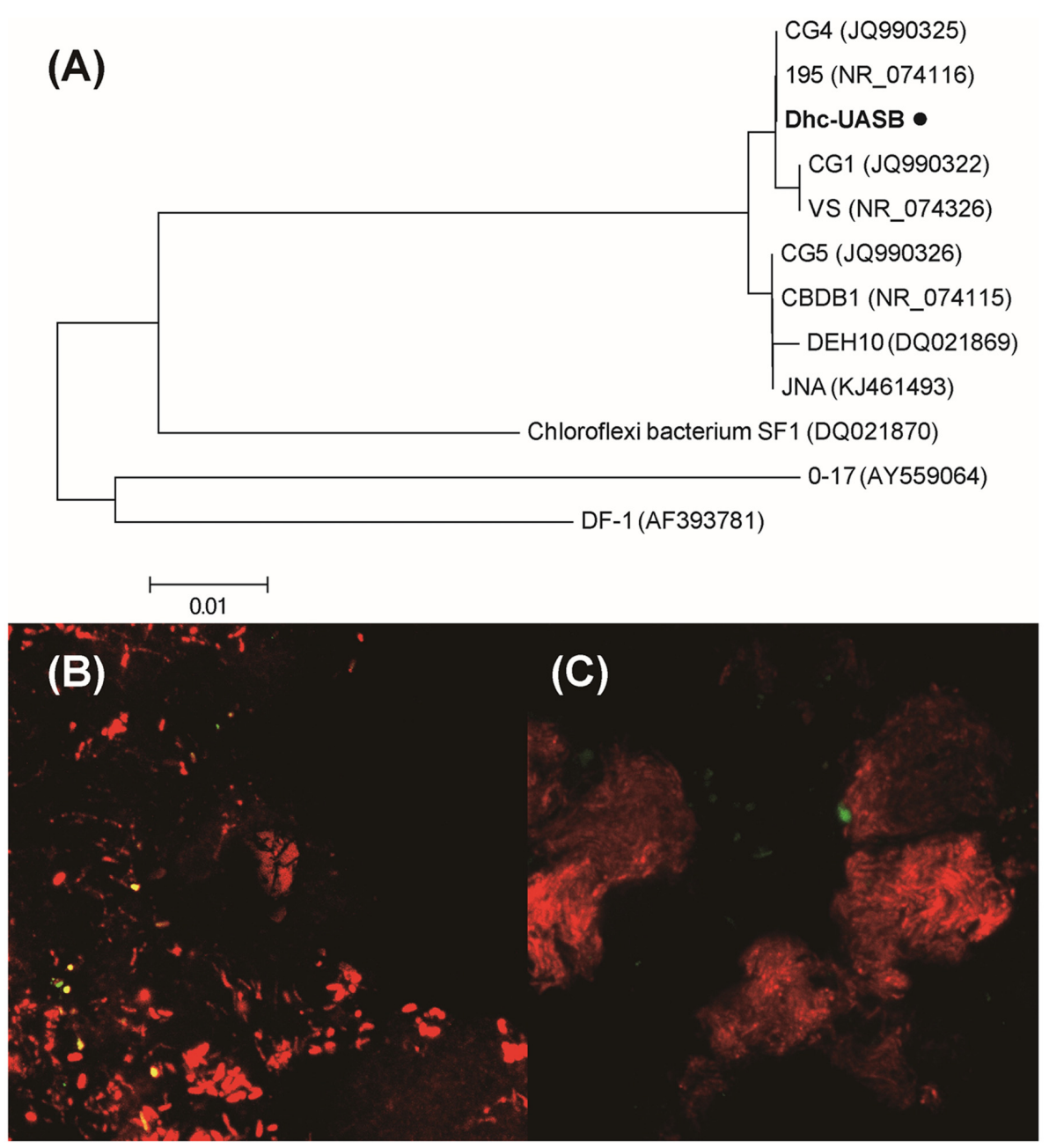

FIGURE 4 | (A) Phylogenetic tree of Dehalococcoides identified in TA-degrading anaerobic granular sludge. Phylogenetic tree was calculated by neighbor-joining method using MEGA4 (Tamura et al., 2007). FISH analysis revealed the space distribution of (B) bacteria (red) and Dehalococcoides (yellow), and (C) archaea (red) and Dehalococcoides (green).

a noteless synthesis byproduct in the petrochemical wastewater generated from terephthalic acid industry (Tomás et al., 2013). In this study, we reported the abundant presence of obligate organohalide-respiring Dehalococcoides in a full-scale UASB reactor for petrochemical wastewater treatment, and further cultivation experiments suggested the possible contamination of bromomethane in the petrochemical wastewater. Recent studies showed experimental evidences of biosynthesis of aromatic organohalides in nature, which might explain the detection of Dehalococcoides in the three black-odorous urban rivers (Agarwal et al., 2014; El Gamal et al., 2016; Şimsir et al., 2017). Also, Dehalococcoides was detected in various environmental samples contaminated with organohalides, including sludge/sediment collected from anaerobic digesters (Smith et al., 2015) and hyporheic zone of a wastewater treatment plant (WWTP)-impacted eutrophic river (Atashgahi et al., 2015). Therefore, Dehalococcoides might be a promising biomarker, complementing current chromatography-based methods, to test organohalide compounds in wastewater and environmental samples.

The UASB reactors provided ideal ecological niches for the growth of Dehalococcoides which further formed syntrophic interactions, as methanogens in syntrophic methanogenic communities (Stams and Plugge, 2009), with aromaticcompound degrading bacteria to overcome the thermodynamic limit through consuming acetate and $\mathrm{H}_{2}$. To our knowledge, this is the first report of the strictly organohalide-respiring Dehalococcoides present abundantly in a full-scale bioreactor for industrial wastewater treatment. In previous studies, Dehalococcoides was documented in various lab-scale bioreactors, including membrane biofilm reactors (Chung et al., 2008), UASB reactor (Hwu and $\mathrm{Lu}, 2008$ ) and anaerobic biotrickling filter (Popat and Deshusses, 2009). The presence of Dehalococcoides in high abundance in both full- and lab-scale bioreactors showed the feasibility of removing toxic and persistent organohalides from various industrial wastewaters in anaerobic bioreactors 
through employing the microbial reductive dehalogenation process.

\section{AUTHOR CONTRIBUTIONS}

SW and DL conceived the idea. QL and LY performed the experiments and data analysis. SW, QY, TL, and ZH provided materials. QL, LY, and SW wrote the manuscript with inputs from all authors. All authors read and approved the final manuscript.

\section{REFERENCES}

Adrian, L., Hansen, S. K., Fung, J. M., Görisch, H., and Zinder, S. H. (2007). Growth of Dehalococcoides strains with chlorophenols as electron acceptors. Environ. Sci. Technol. 41, 2318-2323. doi: 10.1021/es062076m

Adrian, L., Szewzyk, U., Wecke, J., and Görisch, H. (2000). Bacterial dehalorespiration with chlorinated benzenes. Nature 408, 580-583. doi: 10.1038/35046063

Agarwal, V., El Gamal, A. A., Yamanaka, K., Poth, D., Kersten, R. D., Schorn, M., et al. (2014). Biosynthesis of polybrominated aromatic organic compounds by marine bacteria. Nat. Chem. Biol. 10, 640-647. doi: 10.1038/nchembio.1564

Amann, R. I., Ludwig, W., and Schleifer, K. H. (1995). Phylogenetic identification and in situ detection of individual microbial cells without cultivation. Microbiol. Rev. 59, 143-169.

Atashgahi, S., Aydin, R., Dimitrov, M. R., Sipkema, D., Hamonts, K., Lahti, L., et al. (2015). Impact of a wastewater treatment plant on microbial community composition and function in a hyporheic zone of a eutrophic river. Sci. Rep. 5:17284. doi: 10.1038/srep17284

Bedard, D. L., Ritalahti, K. M., and Löffler, F. E. (2007). The Dehalococcoides population in sediment-free mixed cultures metabolically dechlorinates the commercial polychlorinated biphenyl mixture aroclor 1260. Appl. Environ. Microbiol. 73, 2513-2521. doi: 10.1128/AEM.02909-06

Caporaso, J. G., Kuczynski, J., Stombaugh, J., Bittinger, K., Bushman, F. D. Costello, E. K., et al. (2010). QIIME allows analysis of high-throughput community sequencing data. Nat. Methods 7, 335-336. doi: 10.1038/nmeth. f.303

Chu, S., and Hong, C. S. (2004). Retention indexes for temperature-programmed gas chromatography of polychlorinated biphenyls. Anal. Chem. 76, 5486-5497. doi: $10.1021 / \mathrm{ac} 049526 \mathrm{i}$

Chung, J., Krajmalnik-Brown, R., and Rittmann, B. E. (2008). Bioreduction of trichloroethene using a hydrogen-based membrane biofilm reactor. Environ. Sci. Technol. 42, 477-483. doi: 10.1021/es702422d

El Gamal, A., Agarwal, V., Rahman, I., and Moore, B. S. (2016). Enzymatic reductive dehalogenation controls the biosynthesis of marine bacterial pyrroles. J. Am. Chem. Soc. 138, 13167-13170. doi: 10.1021/jacs.6b08512

Elder, D. P., Lipczynski, A. M., and Teasdale, A. (2008). Control and analysis of alkyl and benzyl halides and other related reactive organohalides as potential genotoxic impurities in active pharmaceutical ingredients (APIs). J. Pharm. Biomed. Anal. 48, 497-507. doi: 10.1016/j.jpba.2008.06.009

Fennell, D. E., Nijenhuis, I., Wilson, S. F., Zinder, S. H., and Häggblom, M. M. (2004). Dehalococcoides ethenogenes strain 195 reductively dechlorinates diverse chlorinated aromatic pollutants. Environ. Sci. Technol. 38, 2075-2081. doi: 10.1021/es034989b

He, J., Ritalahti, K. M., Yang, K. L., Koenigsberg, S. S., and Löffler, F. E. (2003). Detoxification of vinyl chloride to ethene coupled to growth of an anaerobic bacterium. Nature 424, 62-65. doi: 10.1038/nature01717

He, J., Robrock, K. R., and Alvarez-Cohen, L. (2006). Microbial reductive debromination of polybrominated diphenyl ethers (PBDEs). Environ. Sci. Technol. 40, 4429-4434. doi: 10.1021/es052508d

Hendrickson, E. R., Payne, J. A., Young, R. M., Starr, M. G., Perry, M. P., and Fahnestock, S. (2002). Molecular analysis of Dehalococcoides 16S ribosomal DNA from chloroethene contaminated sites throughout North America and Europe. Appl. Environ. Microbiol. 68, 485-495. doi: 10.1128/AEM.68.2.485-495. 2002

\section{FUNDING}

This study was supported by the National Natural Science Foundation of China (41671310) and the Key Program of National Natural Science Foundation of China (51638005).

\section{ACKNOWLEDGMENT}

We are very grateful to Feng Yan for providing sludge samples.

Holliger, C., and Schumacher, W. (1994). Reductive dehalogenation as a respiratory process. Antonie Van Leeuwenhoek 66, 239-246. doi: 10.1007/BF00871642

Hwu, C. S., and Lu, C. J. (2008). Continuous dechlorination of tetrachloroethene in an upflow anaerobic sludge blanket reactor. Biotechnol. Lett. 30, 1589-1593. doi: 10.1007/s10529-008-9738-x

Jugder, B., Ertan, H., Bohl, S., Lee, M., Marquis, C. P., and Manefield, M. (2016) Organohalide respiring bacteria and reductive dehalogenases: key tools in organohalide bioremediation. Front. Microbiol. 7:249. doi: 10.3389/fmicb.2016. 00249

Lee, P. K., Men, Y., Wang, S., He, J., and Alvarez-Cohen, L. (2015). Development of a fluorescence-activated cell sorting method coupled with whole genome amplification to analyze minority and trace Dehalococcoides genomes in microbial communities. Environ. Sci. Technol. 49, 1585-1593. doi: 10.1021/ es503888y

Löffler, F. E., Yan, J., Ritalahti, K. M., Adrian, L., Edwards, E. A., Konstantinidis, K. T., et al. (2013). Dehalococcoides mccartyi gen. nov., sp. nov., obligate organohalide-respiring, anaerobic bacteria, relevant to halogen cycling and bioremediation, belong to a novel bacterial class, Dehalococcoidetes classis nov., within the phylum Chloroflexi. Int. J. Syst. Evol. Microbiol. 63, 625-635. doi: 10.1099/ijs.0.034926-0

Lookman, R., Bastiaens, L., Borremans, B., Maesen, M., Gemoets, J., and Diels, L. (2004). Batch-test study on the dechlorination of 1,1,1-trichloroethane in contaminated aquifer material by zero-vaalent iron. J. Contam. Hydrol. 74, 133-144. doi: 10.1016/j.jconhyd.2004.02.007

Lu, Q., Toledo, R. A., Xie, F., Li, J., and Shim, H. (2017). Reutilization of waste scrap tyre as the immobilization matrix for the enhanced bioremoval of a monoaromatic hydrocarbons, methyl tert-butyl ether, and chlorinated ethenes mixture from water. Sci. Total Environ. 583, 88-96. doi: 10.1016/j.scitotenv. 2017.01.025

Lykidis, A., Chen, C. L., Tringe, S. G., McHardy, A. C., Copeland, A., Kyrpides, N. C., et al. (2011). Multiple syntrophic interactions in a terephthalatedegrading methanogenic consortium. ISME J. 5, 122-130. doi: 10.1038/ismej. 2010.125

Mao, X., Stenuit, B., Polasko, A., and Alvarez-Cohen, L. (2015). Efficient metabolic exchange and electron transfer within a syntrophic trichloroethene-degrading coculture of Dehalococcoides mccartyi 195 and Syntrophomonas wolfei. Appl. Environ. Microbiol. 81, 2015-2024. doi: 10.1128/AEM.03464-14

Maymó-Gatell, X., Chien, Y.-T., Gossett, J. M., and Zinder, S. H. (1997). Isolation of a bacterium that reductively dechlorinates tetrachloroethene to ethene. Science 276, 1568-1571. doi: 10.1126/science.276.5318.1568

Mohn, W. W., and Tiedje, J. M. (1992). Microbial reductive dehalogenation. Microbiol. Rev. 56, 482-507.

Müller, J. A., Rosner, B. M., Von Abendroth, G., Meshulam-Simon, G., McCarty, P. L., and Spormann, A. M. (2004). Molecular identification of the catabolic vinyl chloride reductase from Dehalococcoides sp. strain VS and its environmental distribution. Appl. Environ. Microbiol. 70, 4880-4888. doi: 10.1128/AEM.70.8.4880-4888.2004

Narihiro, T., Nobu, M. K., Kim, N. K., Kamagata, Y., and Liu, W. T. (2015). The nexus of syntrophy-associated microbiota in anaerobic digestion revealed by long-term enrichment and community survey. Environ. Microbiol. 17, 1707-1720. doi: 10.1111/1462-2920.12616

Popat, S. C., and Deshusses, M. A. (2009). Reductive dehalogenation of trichloroethene vapors in an anaerobic biotrickling filter. Environ. Sci. Technol. 43, 7856-7861. doi: 10.1021/es901305x 
Rossi, P., Shani, N., Kohler, F., Imfeld, G., and Holliger, C. (2012). Ecology and biogeography of bacterial communities associated with chloroethenecontaminated aquifers. Front. Microbiol. 3:260. doi: 10.3389/fmicb.2012.00260

Schloss, P. D., Westcott, S. L., Ryabin, T., Hall, J. R., Hartmann, M., Hollister, E. B., et al. (2009). Introducing mothur: open-source, platform-independent, community-supported software for describing and comparing microbial communities. Appl. Environ. Microbiol. 75, 7537-7541. doi: 10.1128/AEM. 01541-09

Smidt, H., and de Vos, W. M. (2004). Anaerobic microbial dehalogenation. Annu. Rev. Microbiol. 58, 43-73. doi: 10.1146/annurev.micro.58.030603.123600

Smith, B. J., Boothe, M. A., Fiddler, B. A., Lozano, T. M., Rahi, R. K., and Krzmarzick, M. J. (2015). Enumeration of organohalide respirers in municipal wastewater anaerobic digesters. Microbiol. Insights 8, 9-14. doi: 10.4137/MBI. S31445

Stams, A. J., and Plugge, C. M. (2009). Electron transfer in syntrophic communities of anaerobic bacteria and archaea. Nat. Rev. Microbiol. 7, 568-577. doi: 10.1038/ nrmicro2166

Stringer, R., and Johnston, P. (2001). Chlorine and the environment: an overview of the chlorine industry. Environ. Sci. Pollut. Res. 8, 146-159. doi: 10.1007/ BF02987309

Şimsir, B., Yan, J., Im, J., Graves, D., and Löffler, F. E. (2017). Natural attenuation in streambed sediment receiving chlorinated solvents from underlying fracture networks. Environ. Sci. Technol. 51, 4821-4830. doi: 10.1021/acs.est.6b05554

Tamura, K., Dudley, J., Nei, M., and Kumar, S. (2007). MEGA4: molecular evolutionary genetics analysis (MEGA) software version 4.0. Mol. Biol. Evol. 24, 1596-1599. doi: 10.1093/molbev/msm092

Taş, N., Van Eekert, M. H., Schraa, G., Zhou, J., De Vos, W. M., and Smidt, H. (2009). Tracking functional guilds: "Dehalococcoides" spp. in European river basins contaminated with hexachlorobenzene. Appl. Environ. Microbiol. 75, 4696-4704. doi: 10.1128/AEM.02829-08

Tomás, R. A., Bordado, J. C., and Gomes, J. F. (2013). p-Xylene oxidation to terephthalic acid: a literature review oriented toward process optimization and development. Chem. Rev. 113, 7421-7469. doi: 10.1021/cr300298j

United States Environmental Protection Agency (2013). List of Priority Pollutants. Available at: http://water.epa.gov/scitech/methods/cwa/pollutants.cfm

van der Zaan, B., Hannes, F., Hoekstra, N., Rijnaarts, H., de Vos, W. M., Smidt, H., et al. (2010). Correlation of Dehalococcoides 16S rRNA and chloroethenereductive dehalogenase genes with geochemical conditions in chloroethenecontaminated groundwater. Appl. Environ. Microbiol. 76, 843-850. doi: 10.1128/AEM.01482-09

Wang, S., Chen, S., Wang, Y., Low, A., Lu, Q., and Qiu, R. (2016). Integration of organohalide-respiring bacteria and nanoscale zero-valent iron (Bio-nZVI-RD): a perfect marriage for the remediation of organohalide pollutants? Biotechnol. Adv. 34, 1384-1395. doi: 10.1016/j.biotechadv.2016. 10.004

Wang, S., Chng, K. R., Wilm, A., Zhao, S., Yang, K. L., Nagarajan, N., et al. (2014). Genomic characterization of three unique Dehalococcoides that respire on persistent polychlorinated biphenyls. Proc. Natl. Acad. Sci. U.S.A. 111, 12103-12108. doi: 10.1073/pnas.140484 5111

Wang, S., and He, J. (2013a). Dechlorination of commercial PCBs and other multiple halogenated compounds by a sediment-free culture containing Dehalococcoides and Dehalobacter. Environ. Sci. Technol. 47, 10526-10534. doi: $10.1021 /$ es4017624

Wang, S., and He, J. (2013b). Phylogenetically distinct bacteria involve extensive dechlorination of aroclor 1260 in sediment-free cultures. PLoS ONE 8:e59178. doi: 10.1371/journal.pone.0059178

Yang, Y., and Zeyer, J. (2003). Specific detection of Dehalococcoides species by fluorescence in situ hybridization with 16S rRNA-targeted oligonucleotide probes. Appl. Environ. Microbiol. 69, 2879-2883. doi: 10.1128/AEM.69.5.28792883.2003

Zanaroli, G., Negroni, A., Häggblom, M. M., and Fava, F. (2015). Microbial dehalogenation of organohalides in marine and estuarine environments. Curr. Opin. Biotechnol. 33, 287-295. doi: 10.1016/j.copbio.2015. 03.013

Zhou, Q. X., Cheng, Y., Zhang, Q. R., and Liang, J. D. (2004). Quantitative analyses of relationships between ecotoxicological effects and combined pollution. Sci. China Ser. C 47, 332-339. doi: 10.1360/ 03 yc0042

Zhou, Q. X., and Song, Y. F. (2004). Principles and Methods of Contaminated Soil Remediation (in Chinese). Beijing: Science Press.

Conflict of Interest Statement: The authors declare that the research was conducted in the absence of any commercial or financial relationships that could be construed as a potential conflict of interest.

The reviewer JL declared a past co-authorship with one of the authors QL to the handling Editor.

Copyright (c) 2017 Lu, Yu, Liang, Yan, He, Luan, Liang and Wang. This is an open-access article distributed under the terms of the Creative Commons Attribution License (CC BY). The use, distribution or reproduction in other forums is permitted, provided the original author(s) or licensor are credited and that the original publication in this journal is cited, in accordance with accepted academic practice. No use, distribution or reproduction is permitted which does not comply with these terms. 